\title{
Análise da dimensão ostensiva e não ostensiva em uma atividade avaliativa envolvendo a álgebra vetorial

\author{
Analysis of the ostensive and non-ostentatious dimension in an \\ evaluative activity involving vector algebra
}

\author{
JANY SANTOS SOUZA GOULART ${ }^{1}$ \\ LUIZ MARCIO SANTOS FARIAS ${ }^{2}$
}

\section{Resumo}

Este texto apresenta um recorte de uma pesquisa de doutorado, em andamento, que assume a Álgebra Vetorial como objeto de estudo. Tal conteúdo está inserido no corpus das ementas de componentes curriculares em que o cerne é a Geometria Analítica, presentes na organização curricular de Cursos de Licenciatura em Matemática. Tomamos com aporte teórico a Teoria Antropológica do Didático-TAD, em que um dos pressupostos se centra nas análises das atividades didáticas matemáticas em torno de uma modelação praxeológica composta por complexos de ostensivos e não ostensivos. Assim, o escopo deste estudo se constitui em analisar algumas tarefas contidas em avaliações que versaram sobre os vetores e ao seguir este direcionamento, admitimos como hipótese preliminar que existem relações entre os "conhecimentos inadequados" ou supostos erros, detectados na correção das avalições, e os recursos ostensivos utilizados nas resoluções das questões. Em termos metodológicos, esse estudo servirá para compor parte das análises prévias de uma Engenharia Didática.

Palavras-chave: Teoria Antropológica do Didático. Ostensivos e não ostensivos. Álgebra Vetorial. Avaliação.

\begin{abstract}
This text presents a cut of a doctoral research, in progress that takes as object of study the vector algebra. This content is inserted in the corpus of the curricular components in which the core is the analytical geometry, present in the curricular organization of undergraduate courses in mathematics. We take as theoretical contribution the anthropological theory of didactic-ATD, in which one of the assumptions focuses on the analysis of mathematical didactic activities around praxeological modeling composed of ostensive and non-ostensive complexes. Thus, the scope of this study consists of analyzing some of the tasks contained in evaluations about vectors and following this direction, we assume as preliminary hypothesis that there are relations between "inadequate knowledge" or supposed errors detected in the evaluation of the evaluations, and the
\end{abstract}

\footnotetext{
${ }^{1}$ Universidade Estadual de Feira de Santana- UEFS, Brasil, jany.uefs@gmail.com

${ }^{2}$ Universidade Federal da Bahia - UFBA, Brasil, lmsfarias@ufba.br
} 
ostensible resources used in the resolution of the issues. In methodological terms, this study will serve as part of the previous analysis of a didactic engineering.

keywords: anthropological theory of didactics. Ostensitive and non-ostensive, vector algebra, evaluation.

\section{Résumé}

Ce texte présente une coupe d'une recherche doctorale, en cours qui prend comme objet d'étude l'algèbre vectorielle. Un tel contenu est inséré dans le corpus des menus des éléments du programme dans lequel le noyau est présente la géométrie analytique dans l'organisation des programmes de diplôme en cours de mathématiques. Nous prenons comme base théorique la théorie anthropologique du Didactique dans laquelle l'une des conditions se concentre Si l'analyse des activités d'enseignement mathématiques autour d'un complexe formant praxéologiques consistant manifeste et non ostentatoire. Ainsi, la portée de cette étude est d'analyser certaines tâches contenues dans les évaluations qui traitent avec des vecteurs et suivant cette direction, on admet comme hypothèse préliminaire qu'il existe des relations entre la " connaissance insuffisante » ou des erreurs supposées sur la l'évaluation des évaluations et les ressources ostensibles utilisées pour résoudre les problèmes. En termes méthodologiques, cette étude servira dans le cadre de l'analyse précédente d'une ingénierie didactique.

Mots Clés : théorie anthropologique de la didactique. Ostensive et non-ostensive, algèbre vectorielle, évaluation.

\section{Resumen}

Este texto presenta un recorte de una investigación de doctorado, en marcha, que asume como objeto de estudio el Álgebra Vectorial. Este contenido está insertado en el corpus de los menús de componentes curriculares en que el núcleo es la Geometría Analítica, presentes en la organización curricular de Cursos de Licenciatura en Matemáticas. Tomamos con aporte teórico la Teoría Antropológica del Didáctico - TAD (CHEVALLARD, 1999), en que uno de los presupuestos se centra en los análisis de las actividades didácticas matemáticas en torno a una modelación praxeológica compuesta por complejos de ostensivos y no ostensivos. Por lo tanto, el alcance de este estudio se constituye en analizar algunas tareas contenidas en evaluaciones que versaron sobre los vectores y al seguir este direccionamiento, admitimos como hipótesis preliminar que existen relaciones entre los "conocimientos inadecuados" (BROUSSEAU, 2009) o supuestos errores, corrección de las evaluaciones, y los recursos ostensibles utilizados en las resoluciones de las cuestiones. En términos metodológicos, este estudio servirá para formar parte de los análisis previos de una Ingeniería Didáctica.

Palabras clave: Teoría Antropológica del Didáctico. Ostensivos y no ostensivos. Álgebra vectorial. Evaluación.

\section{Introdução}

As grandezas vetoriais são protagonistas em cursos vinculados às ciências exatas e tecnológicas, compondo, em geral, a ementa de disciplinas em que o cerne é a Geometria 
Analítica e adquire um formato sequencial e recorrente em componentes ligadas a Álgebra Linear, física ou Cálculo. Essa ramificação da abordagem vetorial também está presente e é parte constituinte da estruturação dos Cursos de Licenciatura em Matemática. Aspecto este, que ratifica a relevância da álgebra vetorial dos vetores em cursos de formação de professores de matemática.

No ensino médio e nos cursos básicos universitários, o primeiro contato com os vetores reporta a uma representação em que tais elementos são associados as grandezas que necessitam de módulo, direção e sentido para serem plenamente caracterizadas como em exemplos clássicos de deslocamento, velocidade, aceleração e força dentre outros. Fato este, que remete e retrata a existência de inter-relações com alguns elementos da Geometria Euclidiana (pontos, segmentos de reta, reta, plano, etc.) e o conceito de vetor. A definição de vetor está diretamente associada à relação de equipolência entre segmentos. Conforme apresentado por (STEINBRUCH e WINTERLE, 1987, p.3), dois segmentos orientados $A B e C D$ são equipolentes quando têm a mesma direção, o mesmo sentido e o mesmo comprimento.

Neste aspecto, ao definir vetor determinado por um segmento orientado $A B$ como o conjunto de todos os segmentos orientados equipolentes a $A B$ e indicar como $\vec{v}$ este conjunto, descrito simbolicamente por $\vec{v}=\{X Y / X Y \sim A B\}$ onde $X Y$ é um segmento qualquer do conjunto (STEINBRUCH e WINTERLE, 1987, p.4), emergem uma quantidade significativa de elementos não ostensivos e consequentemente ostensivos, como destacado por Almouloud:

\footnotetext{
A notação $\vec{v}$ e a palavra "vetor", por exemplo, são objetos ostensivos, enquanto a noção de vetor é um objeto não ostensivo, pois é impossível manipulá-lo, contudo podemos torná-lo presente pela manipulação de certos objetos ostensivos a ele associado (ALMOULOUD, 2007, p.120)
}

A título de comparação com a definição anterior de equipolência entres segmentos, apresentamos uma segunda formulação deste conceito retirada de outra obra pertencente às referências bibliográficas da maioria das ementas dos cursos de Geometria Analítica:

Os segmentos orientados $\mathrm{AB}$ e $\mathrm{CD}$ são equipolentes, e indica-se $\mathrm{AB} \sim \mathrm{CD}$, se um dos casos seguintes ocorrer:

a) Ambos são nulos;

b) Nenhum é nulo, e têm mesmo comprimento, mesma direção e mesmo sentido. (CAMARGO e BOULOS, 1987, p.5) 
Constata-se então, que existe um aspecto incompleto e controverso na primeira definição, pois possibilita levantar o seguinte questionamento: Então o se pode afirmar sobre a noção de equipolência para segmentos nulos? De fato, a condição necessária e suficiente é que os todos os segmentos sejam orientados e não podemos fazer referência a orientação do segmento nulo. Indagação essa, que é sanada na segunda conceituação. Perspectivas como estas, nos induz a refletir sobre os obstáculos ou restrições que podem ser gerados a partir de conhecimentos incompletos ou inadequados (BROUSSEAU, 2009). Com base neste esboço, a nossa proposta de estudo se desenha e ganha forma ao analisar alguns entraves detectados em avalições cujo objetos ostensivos e não ostensivos vinculados a álgebra vetorial possam ser pivô para a constituição de obstáculos didáticos.

\section{Um escopo acerca dos obstáculos}

$\mathrm{Na}$ nossa síntese introdutória, apresentamos uma perspectiva conceitual que pode transformar-se em obstáculo à aprendizagem da álgebra vetorial. Contudo, a depender das escolhas didáticas do professor, esse impasse acerca das duas definições vetoriais podem direcionar os alunos a questionarem o que está posto nos materiais institucionais, o que proporciona um despertar para um posicionamento argumentativo e direciona-os a romper com as visitas às obras. Postura esta, ratificada por Chevallard $(2012$, p. 9) ao sinalizar que o cidadão que pertence ao Paradigma de Questionamento do Mundo deve ser convidado a tornasse procognitivo. ${ }^{3}$

Ao consultar a gênese dos obstáculos, especificamente no campo da didática da matemática, nos defrontamos com a noção de obstáculo epistemológico concebida por Gaston Bachelard e divulgada em sua obra intitulada La Formarion de Pesprit scientifique: contribution à une psychanalyse de Ia connaissance de 1938, traduzida para o português em 1996 e que expõe a seguinte declaração:

[...]em termos de obstáculos que o problema do conhecimento científico deve ser colocado. E não se trata de considerar obstáculos externos, como a complexidade e a fugacidade dos fenômenos, nem de incriminar a fragilidade dos sentidos e do espírito humano: é no âmago do próprio ato de conhecer que aparecem, por uma espécie de imperativo funcional, lentidões e conflitos. É aí que mostraremos causas de estagnação e até de regressão, detectaremos causas de inércia às quais daremos o nome de obstáculos epistemológicos. (BACHELARD,1996, p. 17)

\footnotetext{
${ }^{3}$ The citizen in the new paradigm is therefore called upon to become Herbartian, procognitive, and exoteric.
} 
Diante destas lentidões, estagnações e conflitos Bachelard (1996) destacou que o conhecimento do real pode ser comparado a uma luz que consequentemente projetará algumas sombras. Nunca é imediato e pleno, pois as revelações do real são recorrentes. O real nunca é o que se poderia achar, mas é sempre o que se deveria ter pensado. O que significa dizer que o pensamento empírico se torna claro quando o conjunto de argumentos ficam bem estabelecidos. Também segundo o autor, ao retomar um passado cheio de erros, encontra-se a verdade num autêntico arrependimento intelectual. Em resumo, $\mathrm{o}$ ato de conhecer dá-se contra um conhecimento anterior, destruindo conhecimentos mal estabelecidos, superando o que, no próprio espírito, é obstáculo à espiritualização. Ao direcionarmos a atenção para o âmbito da Didática da Matemática encontramos a seguinte vinculação transpositiva.

\begin{abstract}
A transposição em matemática da noção de obstáculo epistemológico, BACHELARD [1938] pensamento que foi reservado para as ciências experimentais, tornou-se possível e até mesmo necessário pelo desenvolvimento da teoria das situações didáticas nos anos 70. (BROUSSEAU, 1989, p. 1, tradução nossa).
\end{abstract}

Nestes termos, Brousseau destacou a existência de um movimento transpositivo dos obstáculos epistemológicos para o campo da didática por meio da Teoria das Situações Didáticas - TSD, a partir deste momento outras configurações de obstáculos surgem, a exemplo dos obstáculos didáticos os quais centram-se um dos ramos do nosso interesse. O mesmo autor, também afirma que:

\footnotetext{
Organizar a superação de um obstáculo consistirá em propor uma situação suscetível de evoluir e de fazer evoluir o aluno, segundo uma dialética conveniente. Não se trata de comunicar as informações que se quer ensinar, mas em encontrar uma situação em que somente elas satisfaçam ou atinjam a obtenção de um resultado satisfatório promovido pelo investimento do aluno (BROUSSEAU, 1976, p. 115, tradução nossa).
}

Em sentido análogo, Almouloud (2007) pontua que o objetivo dessas situações é promover a evolução de concepções inadequadas dos alunos, além de revelar suas concepções espontâneas diante de problemas em que certo conceito possa ser mobilizado. Nas palavras de Brousseau (1976, p. 112, tradução nossa) os obstáculos de origem didática são aqueles que parecem depender apenas de uma escolha ou de um projeto do sistema educativo. O que segundo Almouloud: 
questionável ou incompletos que, mais tarde, revelar-se-ão como obstáculos ao desenvolvimento da conceituação. (ALMOULOUD, 2007, p. 141)

Diante destas constatações, compactuamos com Brousseau (1976) ao admitir que todas concepções são obstáculos a aquisições futuras. Aspecto este que será revelado por meio da análise de duas questões extraída de duas avaliações em que álgebra vetorial constituiu o assunto principal. Assim, que os obstáculos didáticos estabelecerão íntima relação e serão manifestados por meio dos objetos ostensivos que projetarão concepções não ostensivas que provocarão estagnações.

\section{Um olhar acerca das dimensões ostensiva e não ostensiva em atividades que envolvem a álgebra vetorial}

Ao admitir que a aprendizagem matemática extrapola a esfera conceitual, emerge a necessidade de analisar a dupla atribuição assumida pelos ostensivos e não ostensivos nas atividades matemáticas. Elementos estes, que ativam as organizações matemáticas, através da manipulação ostensiva que evoca os não ostensivos, ou seja, a manipulação dos objetos ostensivos permite dar forma a estruturação praxeológica presente na Teoria Antropológica do Didático - TAD (CHEVALLARD, 1999).

Bosch e Chevallard (1999) pontuam que: "os ostensivos têm uma dupla função: eles têm um "valor semiótico", ligado ao seu poder de representar objetos não ostensivos, e um "valor instrumental", ou seja, sua função como ferramentas de práticas matemáticas." (tradução nossa)

Soma-se a essa concepção, o que sinaliza Arzarello, et.al. (2008), que a dimensão ostensiva da atividade matemática é interessante na medida em que possibilita lançar luz sobre problemas de matemática. O que nos leva a considerar que não podemos desprezar as conexões existentes entre as particularidades numéricas, algébricas e geométricas, as quais fazem parte e estão intimamente ligadas às concepções acerca dos vetores.

Assim, a partir deste limiar, revela-se a relevância dos recursos ostensivos como uma espécie de materialização do arcabouço conceitual não ostensivos, possibilitando lançar luz sobre os problemas educacionais da matemática. Nestes termos, analisaremos usando a lente teórica da TAD, em especifico as dimensões ostensiva e não ostensiva, a resolução de duas tarefas similares extraídas de avaliações cujo o cerne foram os vetores. 
Figura 1 - Questão 1

2. Dados os vetores $\vec{u}$ e $\vec{v}$ considere os vetores $\vec{x}$ e $\vec{y}$ definidos por

$$
\vec{x}=\vec{u}-2 \vec{v}, \quad \vec{y}=\vec{u}+\vec{v} .
$$

Calcule o comprimento da projeção ortogonal de $\vec{x}$ sobre $\vec{y}$ nos casos abaixo
(a) $(\mathbf{1}, \mathbf{0})$
$\vec{u}=(1,1,0)$ e $\vec{v}=(0,-1,1)$
(b) $(1,0)$
$\vec{u}$ é unitário, $|\vec{v}|=6$ e o ângulo entre $\vec{u}$ e $\vec{v}$ é $60^{\circ}$.

\section{Fonte: acervo dos autores}

Figura 2 - Questão 2

2. Considere os vetores $\vec{u}=(1,0,1), \vec{v}=(1,-2,0)$ e $\vec{w}=(0,1,-1)$. Resolva as equações
(a) $(0,7)$
$x \vec{u}+y \vec{v}+z \vec{w}=(1,3,1), \quad x, y, z \in \mathbb{R}$.
(b) $(0,8)$
$|\vec{u}-n \vec{w}|^{2}=6, \quad n \in \mathbb{R}$.

Fonte: acervo dos autores

As duas tarefas solicitam dos discentes conhecimentos que envolvem as operações entre vetores (soma, subtração, multiplicação de escalar), a definição de comprimento de um vetor e o conceito de produto escalar assim como, suas aplicações para o cálculo de módulo e ângulo. Nota-se que, na questão $1, \vec{x}, \vec{y}$ e $\vec{z}$ são vetores e na questão $2, x, y$ e $z$ são números reais. A aparente contradição, não se confirma, pois, as questões apresentadas são constituintes de avaliações distintas. Contudo, ao observarmos a resolução das referidas tarefas constatamos que os obstáculos ou restrições estabelecem relações com a diferenciação entre número e vetor.

Figura 3 - Resolução da Questão 1

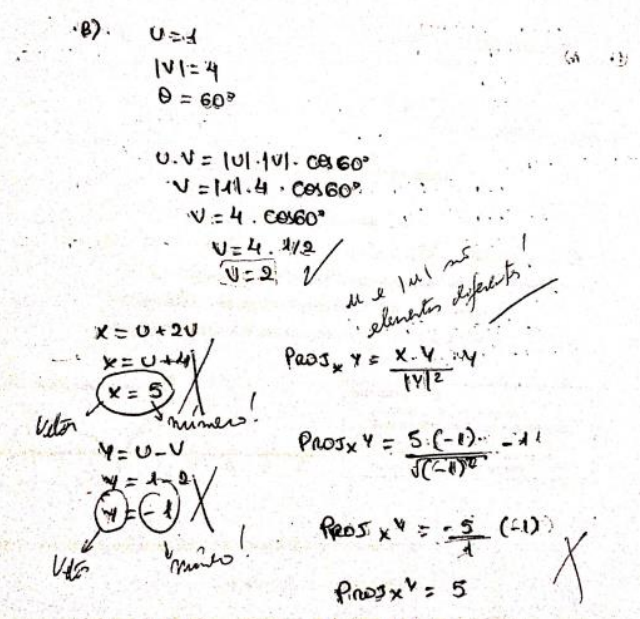

Fonte: acervo dos autores 
A solução apresentada pelo aluno desconsidera o fato que a definição de vetores está intimamente ligada a três caracteristicas básicas (comprimento, direção e sentido) tratando tais elementos como grandezas escalares ao substituir de maneira equivocada, em seus cálculos, o vetor pelo seu comprimento (por exemplo, $y=u-v=1-2=$ -1). Observa-se também que o ângulo entre os vetores $\vec{u}$ e $\vec{v}$ é completamente ignorado na obtenção do comprimento de vetores resultantes da soma e subtração de outros vetores. Ainda que se tenha utilizado o ângulo para obter produto escalar entre $\vec{u}$ e $\vec{v}$, o aluno não fez qualquer uso posterior deste valor.

Figura 4 - Resolução da Questão 2

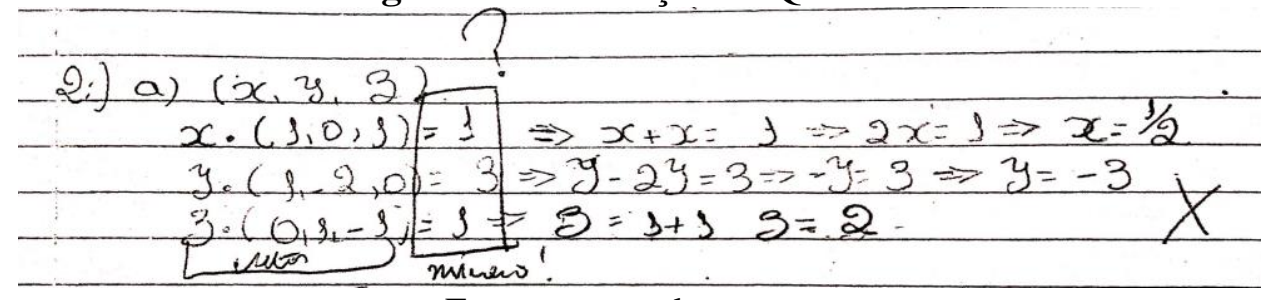

Fonte: acervo dos autores

Nesta resolução, o aluno deixa claro que não compreendeu operações elementares vinculadas ao âmbito teórico da álgebra vetorial ao não conseguir utilizar conceitos como adição, multiplicação de um vetor por um número real e igualdade para construir um sistema linear e, em seguida, obter a solução da questão. Destaca-se ainda que não há distinção entre os conceitos de vetor e número real apresentando, possivelmente, uma tentativa de calcular um produto escalar ao realizar, incorretamente, cálculos como $x$. $(1,0,1)=x+x$.

Não podemos desconsiderar que uso de letras para representar números é abordado inicialmente a partir do sétimo ano do ensino fundamental por meio das expressões numéricas. Perspectiva esta que se propaga por todas os anos subsequentes do ensino fundamental e médio. Como evidenciado na Base Nacional Comum Curricular - BNCC:

No Ensino Fundamental - Anos Finais, os estudos de Álgebra retomam, aprofundam e ampliam o que foi trabalhado no Ensino Fundamental - Anos Iniciais. Nessa fase, os alunos devem compreender os diferentes significados das variáveis numéricas em uma expressão, estabelecer uma generalização de uma propriedade, investigar a regularidade de uma sequência numérica, indicar um valor desconhecido em uma sentença algébrica e estabelecer a variação entre duas grandezas. É necessário, portanto, que os alunos estabeleçam conexões entre variável e função e entre incógnita e equação. As técnicas de resolução de equações e inequações, inclusive no plano cartesiano, devem ser desenvolvidas como uma maneira de representar e resolver determinados tipos de problema, e não como objetos de estudo em si mesmos. (BNCC, 2017, p.269-270) 
Ao analisar as duas resoluções apresentadas acima, notamos que a linguagem algébrica pode constituir um obstáculo para a compreensão dos vetores, revelado pelo equívoco ao igualar número e vetor, destacado na correção do professor. A partir de concepção como estas compactuamos com o questionamento de Brousseau:

\footnotetext{
Deveríamos então pensar que todas as concepções são obstáculos a aquisições futuras? É claro, porque está na sua natureza, como já vimos. Mas muito poucos apresentam dificuldades suficientemente importantes e comuns para serem tratadas como tais. É fácil, todavia, compreender como um super aprendizado precoce pode aumentar as chances de transformar um saber necessário em obstáculo intransponível. (BROUSSEAU, 1976, p.54, tradução nossa)
}

Diante do exposto, nota-se a existência de uma transposição abrupta, ao trata-se de um objeto ostensivo matemático, representado pelas variáveis, incógnitas que expõe generalizações do que foi trabalhado nos anos iniciais do Ensino Fundamental que percorre uma parte significativa da vida escolar dos alunos. São utilizados os mesmos elementos ostensivos como por exemplo $x, y e z$ para manipulação de diferentes não ostensivos, revelando a existência de obstáculos, cuja gênese assenta-se, possivelmente, no primeiro contato que os alunos têm com está representação ostensiva.

\section{Considerações Finais}

Os vínculos estabelecidos entre elementos ostensivos, não ostensivos e os obstáculos didáticos tornam-se evidentes quando confrontamos a gêneses das variáveis ou incógnitas utilizadas nas primeiras generalizações algébricas pertencentes aos últimos níveis do ensino fundamental e sua propagação representacional aparentemente "imutável" em níveis mais avançados, a exemplo dos níveis médio e superior. Vertente esta, ao qual inferimos como uma das possíveis causas de estagnações na aprendizagem da álgebra vetorial.

Outro aspecto que merece destaque, são os registros ostensivos nas ideias matemáticas exibido no desenvolvimento das tarefas vetoriais o que revela formas de manipulações dos objetos ostensivos responsáveis pela estruturação praxeológica na resolução das referidas tarefas. O que apresenta o valor instrumental e semiótico atribuído a dimensão ostensiva da atividade matemática. 
É relevante destacar que a análise apresentada neste artigo, revela algumas restrições que integrarão as análises preliminares e a priori da Engenharia Didática cujo o cerne constituirá na construção e elaboração de situações didáticas que contemplem uma abordagem diferenciada tanto para o ensino quanto para a aprendizagem da álgebra vetorial.

\section{Referências}

ALMOUlOUD, Saddo Ag. Fundamentos da Didática da Matemática. Curitiba: Editora da UFPR, 2007.

ARZARELLO, F.; BOSCH, M.; GASCÓN, J. e SABENA, C. The ostensive dimension through the lenses of two didactic approaches. ZDM Mathematics Education.40:179188, 2008. Disponível em: < https://www.researchgate.net/publication/225589006> Acesso em: 25 de marc. 2018.

BACHELARD, Gaston. A formação do espírito científico: contribuição para uma psicanálise do conhecimento / Gaston Bachelard; tradução Esteia dos Santos Abreu. Rio de Janeiro: Contraponto, 1996. Disponível em: <http://astro.if.ufrgs.br/fis2008/Bachelard1996.pdf> Acesso: 15 de abr. 2018.

BRASIL. Ministério da Educação. Secretaria da Educação Básica. Base Nacional Comum Curricular. Brasília - DF, 2017. Disponível em: < http://basenacionalcomum.mec.gov.br/wp-content/uploads/2018/02/bncc-20dez-site.pdf >. Acesso em: 28 de mai. 2018.

BROUSSEAU, Guy. Les obstacles épistémologiques et la didactique des mathématiques. Nadine Bednarz, Catherine Garnier. Construction des savoirs Obstacles et Conflits, CIRADE Les éditions Agence d'Arc inc., pp.41-63, 1989. Disponível em: < https://hal.archives-

ouvertes.fr/file/index/docid/516581/filename/Les_obstacles_epistemologiques_et_la_di dactique_des_mathematiques89.pdf >. Acesso: 23 de mai. 2018.

Les obstacles épistémologiques et les problèmes en mathématiques. Willy Vanhamme et Jacqueline Vanhamme. La problématique et l'enseignement de la mathématique. Comptes rendus de la XXVIIIe rencontre organisée par la Commission Internationale pour l'Etude et l'Amélioration de l'Enseignement des Mathématiques, Louvain-la-neuve, pp.101-117, 1976. Disponível em: < https://hal.archives-ouvertes.fr/hal-00516569v2/document>. Acesso: 23 de mai. 2018.

Estudos Experimentais e Teóricos de Situações Didáticas em Matemática. In: Curso da Escola de Altos Estudos - EAE. Universidade Bandeirante de São Paulo UNIBAN, 2009. Disponível em: <www.youtube.com/watch?v=BQpdY1P4OM\&t=295s > . Acesso em: 05 abr. 2018.

CAMARGO, Ivan; BOUlOS, Paulo. Geometria Analítica. 3. ed. São Paulo: Prentice Hall, 2005 
CHEVALLARD, Yves; BOSCH, Marianna. La sensibilité de l'activité mathématique aux ostensifs. 1999. Disponível em: <http://yves.chevallard.free.fr/spip/spip/IMG/pdf/ Sensibilite_aux_ostensifs.pdf $>$. Acesso em: 24 abr. 2018.

Teaching Mathematics in Tomorrow's Society: a Case for an Oncoming Counterparadigm. In: INTERNATIONAL CONGRESS ON MATHEMATICAL EDUCATION, 12, 2012, COEX, Seoul, Korea. Anais? Seoul: 2012. Disponível em: <https://link.springer.com/content/pdf/10.1007\%2F978-3-319-12688-3_13.pdf> Acesso: 10 out. 2017.

STEINBRUCH, A.; WINTERLE, P. Geometria Analítica. 2. ed. São Paulo: McGrawHill, 1987. 